\title{
Suppressive Effects of Self-Associated Triblock Copolymers on Quorum Sensing-Mediated Phenazine Antibiotic Production
}

\author{
Chigusa Okano ${ }^{1}$ and Norihiro Kato ${ }^{1,2^{*}}$ \\ ${ }^{1}$ Utsunomiya University, 7-1-2 Yoto, Utsunomiya, Tochigi 321-8585, JAPAN, \\ ${ }^{2}$ CREST, Japan Science and Technology Agency, 7-1-2 Yoto, Utsunomiya, Tochigi 321-8585, JAPAN \\ * Corresponding author: Fax: 81-28-689-6154, e-mail: katon@cc.utsunomiya-u.ac.jp
}

\begin{abstract}
Effective suppression of a bacterial quorum sensing (QS) could be achieved by adding A-B-A tri-block copolymer, poloxamer 84 or 407 into a culture broth, resulting in decrease of the QSmediated phenazine production in Pseudomonas chlororaphis subsp. aurantiaca StFRB508. Dynamic light scattering measurements suggested to form self-associated micelles of respective poloxamer, because a single peak in the size distribution was observed under the same concentration $(1 \mathrm{wt} \%)$ and temperature $\left(30^{\circ} \mathrm{C}\right)$ for the QS suppressive test. The phenazine production in StFRB508 strain was known to be induced by $N$-hexanoylhomoserine lactone (C6HSL) and 3-hydroxy-hexanoylhomoserine lactone (3OH-C6HSL) as QS signaling molecules which can activate an expression of phenazine-related gene after sufficient increase of cell density. The micelles of poloxamer 84 and 407 could suppress the induced phenazine amounts to 55 and $60 \%$, respectively. An $N$-acylhomoserine lactone (AHL) bioassay with Chromobacterium violaceum CV026 suggested effective trap of AHL derivatives to poloxamer micelles because the AHL concentration in the culture broth was obviously reduced in the presence of poloxamers.

Key words: self-associated micelle, triblock copolymer, cell-cell communication, quorum sensing, $\mathrm{N}$-acylhomoserine lactone
\end{abstract}

\section{INTRODUCTION}

Regulation of a bacterial cell-to-cell communication system (QS) is expected as one of preventive strategies for bacterial infection $[1,2]$. Antibiotics possess high ability to suppress the bacterial infection, while its use always involves risks for the appearance of drug resistant bacteria. To minimize the effects of such pathogens, continuous efforts on practical realization of novel antibiotics are necessary. Moreover, a development of novel strategy avoiding the appearance of drug resistant bacteria is also expected. The QS in gram-negative bacteria containing various opportunistic human pathogens such as Pseudomonas aeruginosa and Serratia marcescens is known to be regulated by $\mathrm{N}$-acylhomoserine lactone (AHL) with low molecular weight $[3,4]$. Expression of virulence factors in such pathogens is induced by AHL-mediated QS via the transcriptional activation of the virulence-related genes. The AHLs diffuse in and out of the cells, and accumulate around cells with the cell proliferation. When the AHL concentration reaches a threshold, the AHL and its receptor protein form the complex which can associate with a promoter of the target gene. This means that the increase of the AHL concentration results in the activation of the downstream target gene via the sequential processes [5]. In the case of the $P$. aeruginosa PAO1, $N$-(3-oxododecanoyl)-Lhomoserine lactone (3oxo-C12HSL) produces complex with a LuxR family protein, LasR, which is a homolog receptor of LuxR as the $\mathrm{N}$-(3-oxohexanoyl)-L-homoserine lactone (3oxo-C6HSL) receptor in Vibrio fischeri [6, 7]. The las QS system activates to induce elastase via the AHL-mediated processes $[8,9]$.

Blocking of the AHL-receptor complex formation enables to suppress expression of the pathogenesis-related genes due to keeping the inactive state of the QS. This strategy can prevent infectious diseases without killing the bacteria. Accordingly, we focused on development of holding materials for the AHL, which can reduce the AHL concentration below the threshold even after the cell growth. In the previous studies, cyclodextrin (CD) derivatives [10-12] and liner carboxylated polymers [13] have been studied to capture the AHLs from culture broth of the QS-bacteria. The AHLs are captured respectively by the CDs or the carboxylated polymers through the hydrophobic interaction for the inclusion complex or the binary interactions including hydrophobic interactions and hydrogen bonds. In this research, bio-safe, self-associated poloxamer micelles were investigated to hold AHLs inside the hydrophobic domains because the micelles are developing as one of the expected carriers for the drug delivery into the human body $[14,15]$.

Poloxamers are tri-block copolymers possessing a hydrophobic center domain sandwiched by hydrophilic domains. By hydrophobic interactions of poly(propylene 


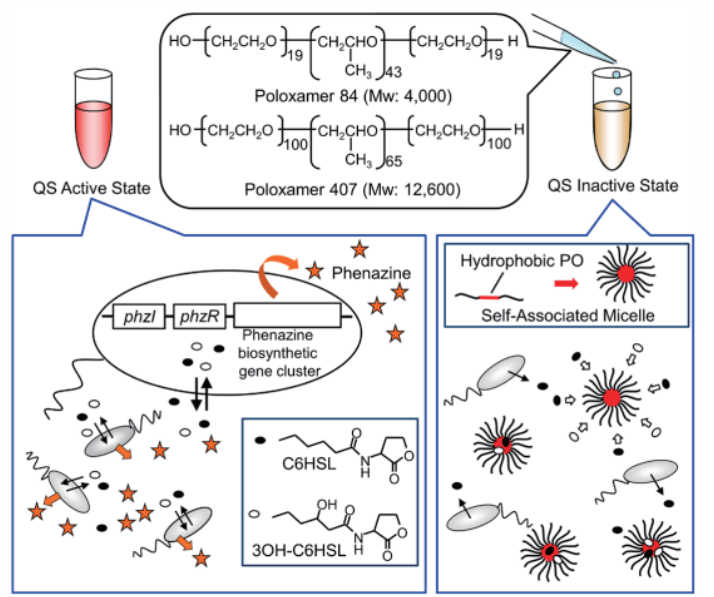

Fig. 1. Suppression of the quorum sensing in the presence of the self-associated poloxamers in Pseudomonas chlororaphis subsp. aurantiaca StFRB508. Phenazine and its derivatives are induced by $N$-acylhomoserine lactone-mediated quorum sensing.

oxide) (PO), the poloxamer molecules can be self-associated to form the micelles possessing hydrophilic shells of poly(ethylene oxide) (EO) $[16,17]$. To investigate effects of the micelle properties on the QS inhibition, two poloxamers with different molecular weight, $\mathrm{EO}_{100}-\mathrm{PO}_{65}$ $\mathrm{EO}_{100}(\mathrm{Mw}: 12,600)$ and $\mathrm{EO}_{19}-\mathrm{PO}_{43}-\mathrm{EO}_{19}(\mathrm{Mw}$ : 4,000), were selected to demonstrate the QS inhibitory effects of Pseudomonas chlororaphis subsp. aurantiaca StFRB508 as model QS bacteria (Fig.1). The StFRB508 strain isolated from a root surface of potato possesses the QS mediated by C6HSL and 3OH-C6HSL. The AHL derived from $p h z I$ which codes the AHL synthase can form the complex with a receptor PhzR to induce production of phenazine and its derivatives as orange antibiotics [18]. As an effective index of the QS activation, the amount of the induced phenazine derivatives is easily determined by visible light adsorption.

In this research, suppressive effects of poloxamers on the AHL-mediated phenazine production in the StFRB508 strain were demonstrated as the QS inhibitory method, and moreover the AHL-poloxamer interaction was discussed using the low molecular poloxamer.

\section{EXPERIMENTAL}

\subsection{Materials and strain}

Poloxamer 407 (Mw: 12,600) and poloxamer 84 (Mw: 4,000) were purchased from Sigma-Aldrich. 8-Anilino-1-naphthalene sulfonic acid (1,8-ANS) was purchased from Kanto chemical. Pseudomonas chlororaphis subsp. aurantiaca StFRB508 was grown in Luria-Bertani (LB) medium for $24 \mathrm{~h}$ at $30^{\circ} \mathrm{C}$ and then stored at $4{ }^{\circ} \mathrm{C}$ until use.

2.2 Characterization of the poloxamers in the LB medium
Particle size distribution of 1 wt $\%$ selfassociated poloxamers in the LB medium was determined by dynamic light scattering, DLS (Nano partica SZ-100Z, Horiba) at 30 or $10^{\circ} \mathrm{C}$. Apparent molecular weight $\left(M_{\mathrm{ap}}\right)$ of the self-associated poloxamers in the LB medium was determined by static light scattering, SLS (SZ-100Z) under the micelle forming conditions (1 wt \% poloxamer, $30^{\circ} \mathrm{C}$ ). Debye plots of both poloxamer 407 and 84 gave straight lines, where a refractive index increment $d n / d C$ ( $n$ : refractive index, $C(\mathrm{mg} / \mathrm{mL})$ : concentration) is set as 0.134 as a representative value of EO in water. The association number of poloxamer molecules in the micelles was estimated from the obtained results and their molecular weight calculated from the chemical formula.

2.3 Hydrophobicity evaluation of the poloxamer dispersed solution by fluorescence measurement

Hydrophobic properties of the dispersed poloxamers in the LB medium are investigated by measuring fluorescence spectra of 1,8-ANS, of which fluorescence intensities increases in the hydrophobic environments [19, 20]. A fluorescence maximum (Ex. $375 \mathrm{~nm}$ ) appears at around $480 \mathrm{~nm}$ when the 1,8-ANS molecules exist at the hydrophobic field. The emission spectra of $10 \mu \mathrm{M}$ 1,8 -ANS in LB medium were determined in the presence of $0.01-1$ wt $\%$ poloxamer 407 or 84 (FP-620, Jasco). The difference spectra with or without poloxamer was determined to avoid effects of an autofluorescence of the LB medium. By plotting fluorescence intensities at $480 \mathrm{~nm}$ against the added poloxamer concentration, hydrophobic properties of the self-associated poloxamer molecules were investigated.

\subsection{Phenazine production assay}

StFRB508 strain was pre-cultured with shaking in $4 \mathrm{~mL}$ of $\mathrm{LB}$ medium for $6 \mathrm{~h}$ at $30^{\circ} \mathrm{C}$. The turbidity of the culture broth at $600 \mathrm{~nm}\left(O D_{600}\right)$ was measured as an index of cell density. After adjusting the $O D_{600}$ to 0.7 with fresh LB medium, main culture was started in $4 \mathrm{~mL}$ of fresh LB medium with $1 \%$ inoculation $\left(O D_{600}=0.007\right)$ for $10 \mathrm{~h}$ at $30^{\circ} \mathrm{C}$. Suppressive effects of the poloxamers on QS were determined by phenazine production. To disperse the hydrophobic phenazine molecules released to the culture medium, $100 \mu \mathrm{L}$ of $10 \%$ sodium dodecyl sulfate solution was added to a $200-\mu \mathrm{L}$ culture broth with vigorously mixing for $10 \mathrm{~s}$, followed by adding water-saturated buthanol. After $10 \mathrm{~s}$ vortex, the almost all phenazine can be extracted into buthanol. A centrifugation $(13,200 \mathrm{rpm})$ for $5 \mathrm{~min}$ can easily separate the buthanol phase from the water phase as well as separate bacterial cell pellets. The extracted buthanol phase was dispensed into a 96-well plate and its absorbance at $365 \mathrm{~nm}(A b s 365)$ was measured. The phenazine production per cell unit $\left(A b s_{365} / O D_{600}\right)$ was calculated as the useful index for the QS activation. Respective value of $A b s_{365} / O D_{600}$ was 


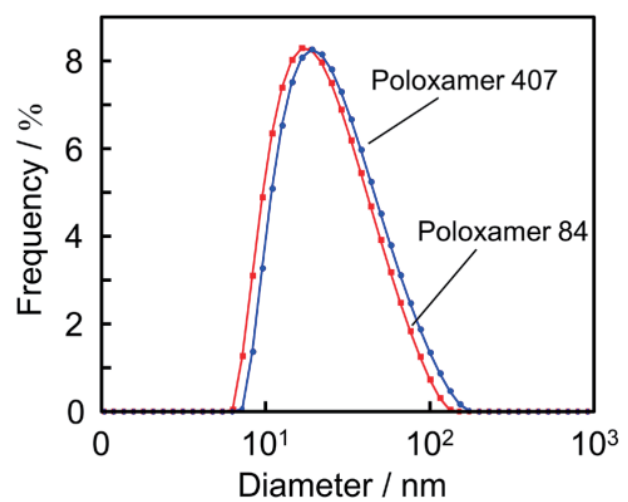

Fig. 2. Size distribution of the poloxamer micelles determined by dynamic light scattering.

normalized by that of the control without any additives.

\subsection{AHL bioassay with Chromobacterium violaceum CV026}

An uptake of the AHL onto the poloxamer micelles was demonstrated by decrease of the AHL concentration in the culture broth using an AHL-synthase gene defective mutant, Chromobacterium violaceum CV026 [21]. A purple pigment, violacein, can be induced by QS activation with additive short-chain AHLs (C4-C8) from outside of cells. The violacein production is induced by 6 pmol AHL as the lower limit [21], and there is a positive correlation between the size of the purple violcein spot appeared on the CV026 agar plate and the AHL concentration of the sample solution. The CV026 was pre-cultured for $10 \mathrm{~h}$ at $30^{\circ} \mathrm{C}$ in 4 $\mathrm{mL}$ LB medium with antibiotic kanamycin. The pre-cultured medium was mixed with $100 \mathrm{~mL}$ of fresh LB agar medium at the sol state as $1 \%$ inoculation. The agar gel plates containing CV026 cells were prepared by pouring the mixture into Petri dishes and then were cooled. After the culture of StFRB508 strain for $10 \mathrm{~h}$ with 0.05 or $1 \mathrm{wt} \%$ poloxamer 407 as the same condition for the QS suppressive test, the culture broth was centrifuged at $7,500 \mathrm{~g}$ for $15 \mathrm{~min}$ with a centrifugal ultrafiltration device of which cutoff molecular weight is $10,000 \mathrm{Da}$ (Amicon Ultra $10 \mathrm{~K})$. Approximately $400 \mu \mathrm{L}$ of the filtrate was vigorously mixed with $400 \mu \mathrm{L}$ of ethyl acetate to extract the organic matter including AHLs into the ethyl acetate phase. The ethyl acetate phase separated by centrifugation $(13,200 \mathrm{rpm}, 5 \mathrm{~min})$ was dried to dryness under reduced pressure, followed by adding $20 \mu \mathrm{L}$ of water-miscible solvent, dimethyl sulfoxide (DMSO). The $15 \mu \mathrm{L}$ of AHL extracts in DMSO was spotted onto a paper disk $(\phi: 8 \mathrm{~mm})$ placed on the CV026 plate. The plate was incubated at $30^{\circ} \mathrm{C}$ for $24 \mathrm{~h}$ to induce the purple violacein spot via the QS activation. As compared with the diameter of the purple spot derived from the control without any poloxamers, appearance of the purple spot with
Table 1 Apparent molecular weights and association numbers of self-associated poloxamers.

\begin{tabular}{l||c|c}
\hline & $M_{\mathrm{ap}} / \mathrm{kDa}$ & $\begin{array}{c}\text { Association } \\
\text { number }\end{array}$ \\
\hline \hline poloxamer 84 & 75.4 & 18.9 \\
\hline poloxamer407 & 126.3 & 10.0 \\
\hline
\end{tabular}

smaller diameter is reflected to the AHL uptake onto the added poloxamers.

\section{RESULTS AND DISCUSSION}

3.1 Characterization of the poloxamers in the LB medium

Under the condition of self-associated micelle formation of A-B-A tri-block copolymers by hydrophobic interaction in water, the size of the poloxamer should be greater than that expected from its molecular weight. The single-peaked size distribution $\left(30^{\circ} \mathrm{C}\right)$ for $1 \mathrm{wt} \%$ of poloxamer 84 and 407 in the LB medium were obtained by DLS measurement in Fig. 2. The mode diameters of the poloxamer 84 and 407 were 15.5 and $17.8 \mathrm{~nm}$, respectively. A critical micelle concentration (CMC) of the poloxamer generally decreases with increasing temperature, where the CMCs of poloxamer 84 and 407 respectively are 0.6 and $0.1 \mathrm{wt} \%$ in water at $30^{\circ} \mathrm{C}$ [22]. Disappearance of the 17.8-nm peak in DLS clearly suggests the dissociation of the micelles after cooling to $10^{\circ} \mathrm{C}$ (data not shown); the CMC of the poloxamer 407 plausibly shifts to below $1 \mathrm{wt} \%$. Apparent molecular weights determined by SLS measurement and association numbers of the poloxamer molecules based on their true molecular weight are summarized in Table 1. As expected, the association number of the low molecular poloxamer $84(\mathrm{Mw}: 4,000)$ was greater than that of the poloxamer $407(\mathrm{Mw}: 12,600)$ due to avoiding steric hindrance. The poloxamer molecules could be self-associated by intermolecular hydrophobic interaction via the hydrophobic PO units. The apparent molecular weights of the hydrophobic PO units per micelle were 47.2 and $37.8 \mathrm{kDa}$ in poloxamer 84 $\left(\mathrm{EO}_{19}-\mathrm{PO}_{43}-\mathrm{EO}_{19}\right)$ and $407\left(\mathrm{EO}_{100}-\mathrm{PO}_{65}-\mathrm{EO}_{100}\right)$, respectively. These results suggested that the low molecular poloxamer 84 could easily form the hydrophobic domain inside the micelle.

The poloxamer micelles are applied to the carriers for hydrophobic drugs because the micelle can be dispersed in aqueous solution via their hydrophilic EO layers [14]. Accordingly, the uptake of the AHLs possessing hydrophobic acyl-chains onto the micelles is expected in the culture medium. Formation of the hydrophobic moiety on the micelle was investigated by emission properties of 1,8-ANS probe of which fluorescence intensity is depending on the polarity around it. To cancel the autofluorescence of the LB medium, the difference spectra were given against the LB medium in the absence of any poloxamers (Fig.3a). The fluorescence intensities of 1,8 -ANS in the LB medium clearly 

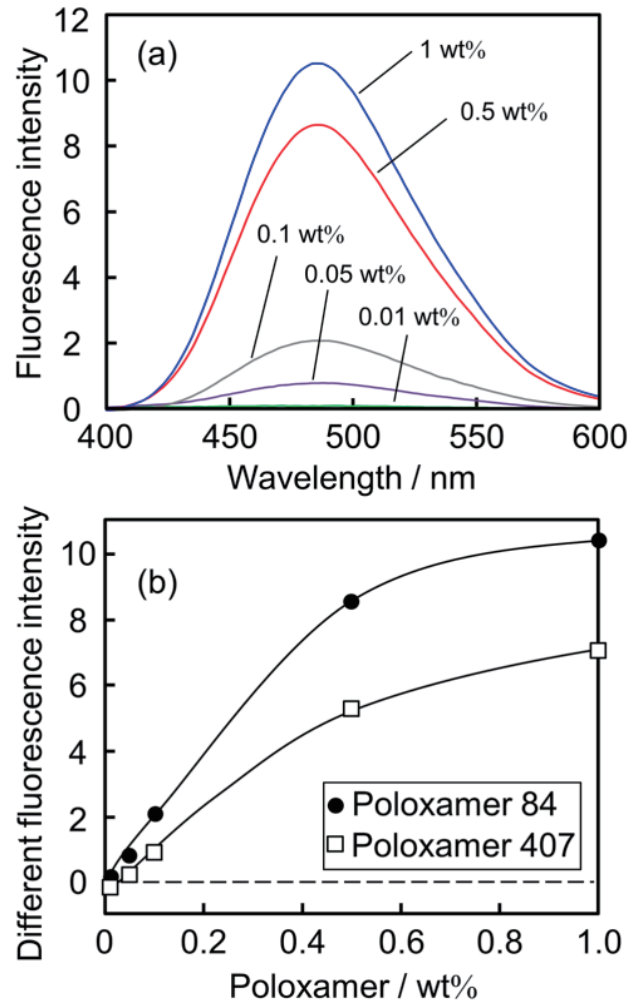

Fig. 3. (a) Different emission spectra of the 1,8-ANS dissolved in the LB medium with 0-1 wt $\%$ poloxamer 84 . (b) Different fluorescence intensity at $480 \mathrm{~nm}$ of the 1,8 -ANS in the LB medium with $0-1$ wt $\%$ poloxamer 84 or 407 .

increased with increasing the poloxamer 84 concentration. The increment of the fluorescence intensity at $480 \mathrm{~nm}$ corresponds to expansion of the hydrophobic moiety in the solution. Figure $3 \mathrm{~b}$ showed the plots of fluorescence intensities at $480 \mathrm{~nm}$ against the concentrations of the poloxamer 407 in addition to the poloxamer 84 . Both intensities increased with increasing the poloxamer concentration and then tended to level off. After the formation of the micelles at above the CMC, the content of the hydrophobic moiety approached to the constant. Furthermore, the poloxamer 84 shows higher fluorescence intensity than that of the poloxamer 407 irrespective of their concentration. This result is probably attributed to the higher content of the PO unit per micelle for the poloxamer 84 as shown in Table 1 .

\subsection{Suppressive properties on QS with poloxamers}

Suppressive effects of poloxamers on the QS-mediated phenazine production in StFRB508 strain are shown in Fig.4. Relative phenazine production $\left(A b_{3365} / O D_{600}\right)$ was normalized by the control value and plotted against the poloxamer concentration. The relative phenazine production for both poloxamers decreased to approximately 0.6 with increasing the poloxamer concentration, whereas the hydrophilic EO (Mw:10,000) showed a smaller effect than the triblock poloxamer. The

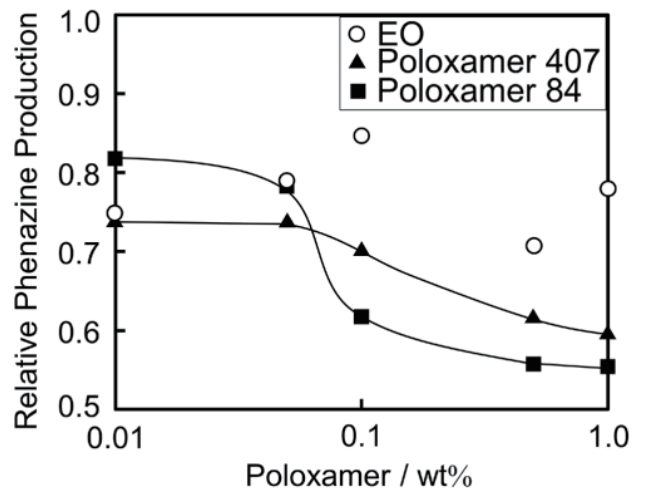

Fig. 4. Suppressive effects of poloxamers or polyethylene glycol (EO) on the QS-mediated phenazine production in $P$. chlororaphis subsp. aurantiaca StFRB508.

results suggested that suppressive effects of the poloxamer 407 on QS improved above its CMC $(0.1 \mathrm{wt} \%)$, whereas the effects of the poloxamer 84 became prominent at around 0.01-0.1 wt\% correspond to below its CMC $(0.6 \mathrm{wt} \%)$. The previous studies clearly showed that the hydrophobic drugs could be held easily on poloxamer micells which were self-associated at high concentration than the CMC [14]. The results suggest the effective uptake of the AHLs on the poloxamer 407 micelles via the hydrophobic interaction in the same manner. On the other hand, the AHLs were suggested to interact with poloxamer 84 molecules in a different principle as compared with the poloxamer 407 molecules. The interpretation is consistent with the results of fluorescence measurements as shown in Fig. $3 \mathrm{~b}$; the expression of the 1,8-ANS fluorescence below the CMC of poloxamer 84 suggested the presence of hydrophobic environments even when not forming micelles. Low-weight molecules even possessing hydrophobic domains can disperse well in aqueous solution as a single or small multimer due to forming the hydration shell. Thus, the hydrophobic acyl-chain of the AHLs plausibly interacts with such associated domain of the hydrophobic PO. Great efforts of the detection on such small multimers were not able to lead to the results because the experimental condition $(0.1$ wt $\%$ poloxamer) was out of the detection limit of DLS.

Another possibility is the interaction derived from a hydrogen bond. Judging from the QS suppressive test as shown in Fig.4, the AHLs enabled to interact with additive low-weight EO molecules (Mw: 10,000), which corresponding to the nearly same size of the EO domain (EO) 200 (Mw: 8,800) in poloxamer 407. In the presence of $0.1 \mathrm{wt} \% \mathrm{EO}$, the relative phenazine production decreased to approximately 0.85 through the QS suppression. As an example, the QS signal, $\mathrm{N}$-(3-oxooctanoyl) homoserine lactone (3oxoC8HSL), produced in a plant pathogen Agrobacterium tumefaciens can interact with their 


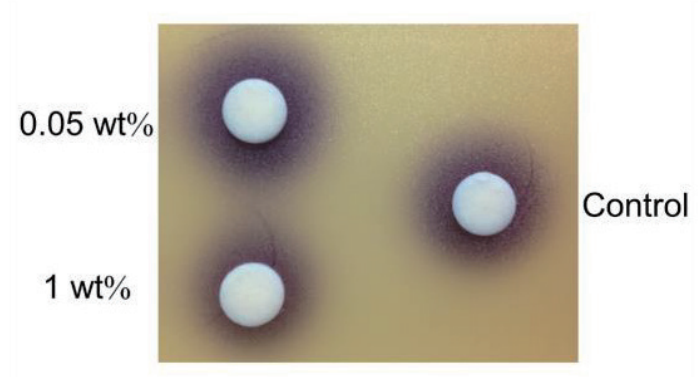

Fig. 5. The AHL bioassay for the supernatant of the StFRB508 culture broth using $C$. violceum CV026. The StFRB508 was grown in the presence of $0,0.05$, or $1 \mathrm{wt} \%$ poloxamer 407. After removing the micelles, the remaining AHLs dissolved in the supernatant could activate the QS-dependent violacein production.

receptor TraR which is a member of the LuxR family proteins [23]. The complex of the TraR with amphiphilic AHLs could be stabilized by hydrogen bonding among Trp57, Asp70, and the homoserine lactone ring, as well as hydrophobic interaction between the AHL acyl chains and some hydrophobic side chains of the amino acid residues $[23,24]$.

3.3 Bioassay analysis of the AHL-poloxamer interaction

The extract solution of AHLs and others from StFRB508 culture was spotted onto a paper disk placed on the CV026 agar plate. The poloxamer associates could be removed from the extract solution by centrifugal ultrafiltration. After $24 \mathrm{~h}$ incubation at $30^{\circ} \mathrm{C}$, the purple shade appeared on the plate clearly indicated the violacein production which could be induced solely by adding AHLs (Fig.5). The shade was observed in concentric circles around the paper disk and its diameter was positively correlated with the AHL content. The AHL extract samples were similarly prepared from the StFRB508 culture in the presence of $0,0.05$, or $1 \mathrm{wt} \%$ poloxamer 407 . The diameter of the purple shade derived from the culture under the condition of $1 \mathrm{wt} \%$ poloxamer 407 was smaller than that of the control, whereas no significant difference was observed between the diameters with or without $0.05 \mathrm{wt} \%$ poloxamer 407. This means that the decrease of the free AHL concentration in the culture broth could become detectable by adding the poloxamer 407 as the same concentration of CMC. The AHL uptake onto the micelles is probably responsible for the decrease of the free AHLs in the culture, which can lead to the QS suppression in $P$. chlororaphis subsp. StFRB508 as shown in Fig. 4.

\section{CONCLUSIONS}

The QS-mediated phenazine production under the control of C6HSL and $3 \mathrm{OH}-\mathrm{C} 6 \mathrm{HSL}$ in $P$. chlororaphis subsp. StFRB508 was suppressed by adding the micelles of Poloxamer 407 or 84 , of which mode diameters were observed as 17.8 or $15.5 \mathrm{~nm}$, respectively. The induced phenazine amount respectively was only 60 or $55 \%$ of the control in the presence of $1 \mathrm{wt} \%$ poloxamer 407 or 84 at $30^{\circ} \mathrm{C}$. The $\mathrm{CV} 026$ bioassay shows that the some of the AHLs dissolved in the culture are able to be captured on the poloxamer 407 micelles. The decrement of the phenazine production suggests that the self-associated poloxamer micelles are useful AHL carriers to capture the AHLs to inhibit the QS in gram-negative bacteria. Small multimers of the low-molecular EO-PO-EO type copolymers probably interact with the AHLs out of the condition on forming micelles, because lowweight poloxamer 84 could obviously suppress the QS at the concentration below the CMC.

\section{ACKNOWLEDGEMENTS}

This work was supported by Core Research for Evolutional Science and Technology-Japan Science and Technology Agency (CREST-JST) and JSPS KAKENHI Grant Number 25.9098.

\section{REFERENCES}

[1] T. R. de Kievit and B. H. Iglewski, Infect. Immun., 68, 4839-4849 (2000).

[2] S. B. Tay and W. S. Yew, Int. J. Mol. Sci., 14, 16570-16599 (2013).

[3] R. S. Smith, S. G. Harris, R. Phipps, and B. Iglewski, J. Bacteriol., 184, 1132-1139 (2002).

[4] H. Slater, M. Crow, L. Everson, and G. P. C. Salmond, Mol. Microbiol., 47, 303-320 (2003).

[5] N. A. Whitehead, A. M. L. Barnard, H. Slater, N. J. L. Simpson, and G. P. C. Salmond, FEMS Microbiol. Rev., 25, 365-404 (2001).

[6] J. Slock, D. van Riet, D. Kolibachuk, and E. P. Greenberg, J. Bacteriol., 172, 3974-3979 (1990).

[7] M. J. Gambello and B. H. Iglewski, J. Bacteriol., 173, 3000-3009 (1991).

[8] P. Cosson, L. Zulianello, O. Join-Lambert, F. Faurisson, L. Gebbie, M. Benghezal, C. van Delden, L. K. Curty, and T. Köhler, J. Bacteriol., 184, 3027-3033 (2002).

[9] R. S. Smith, S. G. Harris, R. Phipps, and B. Iglewski, J. Bacteriol., 184, 1132-1139 (2002).

[10] T. Morohoshi, K. Tokita, S. Ito, Y. Saito, S. Maeda, N. Kato, and T. Ikeda, J. Biosci. Bioeng., 116, 175-179 (2013).

[11] N. Kato, T. Tanaka, S. Nakagawa, T. Morohoshi, K. Hiratani, and T. Ikeda, $J$. Inclusion Phenom. Macrocyclic Chem., 57, 419-423 (2007).

[12] N. Kato, A. Kobayashi, H. Motohashi, Y. Ozonoe, T. Morohoshi, and T. Ikeda, Prog. Colloid Polym. Sci., 136, 155-162 (2009).

[13] E. V. Piletska, G. Stavroulakis, K. Karim, M. J. Whitcombe, I. Chianella, A. Sharma, K. E. Eboigbodin, G. K. Robinson, and S. A. Piletsky, Biomacromol., 11, 975-980 (2010).

[14] A. V. Kabanov, E. V. Batrakova, and V. Y. Alakhov, J. Control. Release, 82, 189-212 (2002). [15] C. Okano and N. Kato, Chem. Lett., 44, 
1544-1546 (2015).

[16] T. H. Vaughn, H. R. Suter, L. G. Lundsted, and M. G. Kramer, J. Am. Oil. Chem. Soc., 28, 294-299 (1951).

[17] R. Nagarajan, Colloids Surf. B: Biointerfaces, 16, 55-72 (1999).

[18] T. Morohoshi, W.-Z. Wang, T. Suto, Y. Saito, S. Ito, N. Someya, and T. Ikeda, J. Biosci. Bioeng., 116, 580-584 (2013).

[19] E. M. Kosower, H. Dodiuk, K. Tanizawa, M. Ottolenghi, and N. Orbach, J. Am. Chem. Soc., 97, 2167-2178 (1975).

[20] E. M. Kosower, H. Dodiuk, and H. Kanety, J. Am. Chem. Soc., 100, 4179-4188 (1978).

[21] L. Ravn, A. B. Christensen, S. Molin, M. Givskov, and L. Gram, J. Microbiol. Methods, 44, 239-251 (2001).

[22] P. Alexandridis, J. F. Holzwarth, and T. A. Hatton, Macromolecules, 27, 2414-2425 (1994).

[23] A. Vannini, C. Volpari, C. Gargioli, E. Muraglia, R. Cortese, R. De Francesco, P. Neddermann, and S. Di Marco, EMBO J., 21, 4393-4401 (2002).

[24] R.-G. Zhang, T. Pappas, J. L. Brace, P. C. Miller, T. Oulmassov, J. M. Molyneaux, J. C. Anderson, J. K. Bashkin, S. C. Winans, and A. Joachimiak, Nature, 417, 971-974 (2002).

(Received October 23, 2015; Accepted November 19, 2015) 